\title{
Un análisis del efecto de la percepción de justicia de precios en el comportamiento del consumidor en el proceso de reserva de hotel online
}

\author{
M $^{\mathrm{a}}$ Encarnación Andrés Martínez* Miguel Ángel Gómez Borja** \\ Juan Antonio Mondéjar Jiménez*** \\ Universidad de Castilla-La Mancha (España)
}

\begin{abstract}
Resumen: La percepción de justicia juega un rol muy importante en la toma de decisiones del consumidor. La situación económica de muchos hogares hace que la percepción de justicia de precios sea crucial en el comportamiento de compra. Esta situación no es ajena a un sector tan importante como el sector turístico, y concretamente a la reserva de hoteles. En este trabajo se considera la reserva de un hotel online para analizar las consecuencias en el comportamiento de compra que se derivan de la percepción de justicia de precios. Éstas se ponen de manifiesto a largo plazo cuando la situación es irreversible, por lo que los vendedores deben conocerlas para tener claros los efectos que puede provocar la consideración de sus precios como injustos.
\end{abstract}

Palabras Clave: Percepción de justicia; precios; satisfacción; lealtad; confianza en la decisión.

An analysis of the effect of the fairness price perception on the consumer behavior: the case of online hotel reservation

Abstract: The fairness perception has an important role in consumer decision. The economic situation of many households makes that the fairness price perception was very important in the purchase behavior. This situation is not alien to such an important sector as the tourism sector, and specifically to the hotel booking. In this paper we consider the online hotel booking to analyze the consequences on purchase behavior stemming from the fairness price perception. These effects can be seen in the long term when the situation is irreversible, therefore the sellers should know those consequences to be clear about the effects that can have if their prices are considered unfair.

Keywords: Fairness perception; price; price satisfaction; loyalty; self-confidence.

\section{Introducción}

Las investigaciones tradicionales de marketing relacionadas con los precios se han centrado fundamentalmente en encontrar los determinantes económicos usados en la fijación de los mismos. Sin embargo, se tiende a enriquecer estos modelos considerando motivos no racionales relacionados con la percepción de precios, ya que un elemento importante a la hora de fijar los precios es analizar si los consumidores consideran ese precio como equitativo y justo.

En justicia de precios, debido a la situación económica que se está atravesando se ha vuelto a evocar, de manera acentuada, el interés por parte del consumidor por conseguir "precios justos". De modo que la crisis económica ha contribuido en algunos casos a aumentar la sensibilidad de los consumidores al precio, haciendo que presten más atención al mismo, así como también hacia la consecución de "mejores

\footnotetext{
* E-mail: encarnacion.andres@uclm.es

** E-mail: miguelangel.gborja@uclm.es

*** E-mail: juanantonio.mondejar@uclm.es
} 
tratos". En este sentido, trabajos como el de Andrés et al. (2012) analizan los principales antecedentes que influyen en la percepción de precios, si bien, son pocos los trabajos que analizan de forma conjunta las distintas consecuencias en el comportamiento de compra que se derivan de una percepción de injusticia por parte del consumidor.

La importancia de la percepción de justicia de precios es evidente para las empresas debido a la influencia que ejerce en el comportamiento de compra del consumidor (Gielissen et al., 2008), ya que tal y como señalan Kahneman et al. (1986 a y b) la justicia percibida en el precio constituye el factor psicológico que más influencia ejerce en el comportamiento del consumidor ante el precio. De ahí, que sea interesante conocer cómo se perciben los precios y qué cambios se perciben como justos o injustos, ya que las valoraciones sobre justicia influyen sobre el comportamiento de elección de los compradores (e.g. influencia sobre satisfacción, lealtad o valoración de los productos y servicios).

La incorporación de Internet como canal de ventas ha producido una nueva forma de comprar donde no se requiere ir a un establecimiento físico para adquirir un producto, sino que se puede comprar desde cualquier lugar. Además, Internet se está convirtiendo en un entorno de interacción que permite realizar comparaciones de precios con gran facilidad, por lo que la percepción de la justicia de los precios adquiere una mayor importancia. Esta mayor relevancia proviene al menos de dos ideas: por un lado, los vendedores disponen de más capacidad para discriminar los precios según la sensibilidad del consumidor a los mismos, y, por otro lado, los consumidores tienen a su disposición herramientas y aplicaciones con las que pueden conseguir información del precio aportada por otros consumidores (e.g. TripAdvisor) o incluso comparar el precio para distintos vendedores. Estos dos aspectos conducen a que se otorgue más relevancia al fenómeno de la justicia en los precios en el canal online (Bolton et al., 2010).

Este trabajo se ha centrado en el sector turístico dado el peso que este sector representa sobre el total del producto interior bruto, concretamente, en 2010 representa el 10,2\% según Hosteltur (2011). Esta relevancia se pone de manifiesto en las compras online, ya que las actividades vinculadas con dicho sector como compras de billetes de transporte (avión, tren,...), así como reservas de alojamiento entre otros se consideran como productos estrella en las adquisiciones online (Observatorio Nacional de las Telecomunicaciones y de la Sociedad de la Información, ONTSI, 2011).

Otros datos que afianzan la importancia de la compra de billetes de transporte y reservas de alojamiento online son los publicados por ONTSI (2011) en los que los billetes de transporte y la reserva de alojamiento con un $52,4 \%$ y un $42,9 \%$ respectivamente constituyen los productos estrella del comercio electrónico. En este mismo sentido, el Instituto Nacional de Estadística 2011 (INE) muestra que durante los últimos meses (octubre 2010- octubre 2011) alojamiento y viajes son los servicios más adquiridos por Internet con un $52,9 \%$ y $49 \%$ del total, respectivamente. Teniendo presente estos argumentos se ha seleccionado, utilizando fuentes de información secundarias como la Encuesta de Ocupación Hotelera (EOH) elaborada por el INE (2012), hoteles de cuatro estrellas dado que son los más demandados por los viajeros

Los argumentos anteriores ponen de manifiesto que el sector turístico en Internet cada vez tiene más presencia en el mismo. En el año 2011 se observa que el canal online es el medio más utilizado para realizar una reserva de alojamiento $(51,4 \%)$, así como para buscar, básicamente, información de alojamientos (75\%), y comparar precios a través de Internet (72,7\%) (ONTSI, 2011).

Así, en la segunda sección se recoge un análisis de las principales consecuencias derivadas de la percepción de justicia de precios por parte del consumidor en Internet y las hipótesis a contrastar en el trabajo. La tercera sección recoge la metodología empleada en la aplicación empírica desarrollada centrándose en la muestra obtenida, las variables utilizadas y los principales resultados obtenidos mediante la estimación de un modelo PLS. Por último, aparecen las principales conclusiones y líneas de investigación futura.

\section{La percepción de justicia de precios y sus principales consecuencias}

La percepción del precio es un estímulo complejo y amplio que consiste en indicaciones positivas y negativas (Lichtenstein et al. 1993). Estas indicaciones positivas se pueden traducir en una valoración de los precios como justos y en el caso de indicaciones negativas como injustos. Los precios que se perciben como injustos influyen de manera negativa en las intenciones de volver a comprar, y además generan desconfianza. Estos efectos tienen lugar tanto en el canal tradicional como en el virtual (Kahneman et al., 1986 a y b; Campbell, 1999), aunque, si cabe, con mayor incidencia en el canal virtual.

Hay que tener en cuenta que dentro del proceso de compra online, según Cao et al. (2003), se pueden distinguir dos fases. En la primera fase, el cliente busca un producto en distintas páginas, después compara 
rasgos, hace una selección y a continuación el pedido. Esto es lo que se conoce como proceso de pedido. En segundo lugar, una vez que se ha realizado el pedido, cuando éste llega al cliente puede quedárselo o devolverlo. Esto es lo que se conoce como proceso de cumplimiento -fulfillment-. En este trabajo nos vamos a centrar en el proceso de pedido ya que aunque el proceso de recogida de la información recoge todo el proceso de compra, no llegamos a valorar la situación una vez realizada la reserva.

Sin duda, el canal en el que se realiza la compra tiene una influencia clara en el proceso de percepción de justicia del precio. En este sentido, Yu (2008) toma como base el estudio realizado por Lichtenstein et al. (1993), que establece que la percepción del precio es distinta según el canal que se utilice; y los compradores online tienden a percibir los precios más altos de forma más negativa que los compradores offline. Esto se debe a que los compradores online son muy conscientes del valor del precio, tienen un nivel más alto de conciencia del precio y propensión a la venta que los compradores offline, así como una mayor probabilidad de adoptar Internet como su canal.

La percepción del precio genera distintas respuestas y reacciones emocionales en el comprador según cuál sea la situación que experimenta (precios justos vs. precios injustos), reacciones que en algunos casos pueden presentar grandes consecuencias para el vendedor. En relación a las consecuencias que se derivan tras la percepción de justicia del precio del consumidor, cabe señalar que la percepción de justicia del precio adquiere gran relevancia, ya que la satisfacción del consumidor y su comportamiento se forman en base a esas percepciones (Oliver y Swan, 1989; Bei y Chiao, 2001). De modo que, la percepción de injusticia del precio influye tanto en la satisfacción del consumidor, como en las intenciones de compra y en las acciones (posibles reclamaciones) desarrolladas contra el vendedor (Campbell, 1999).

\subsection{Percepción de justicia de precios y confianza en la decisión}

La confianza que tiene el consumidor se define como la importancia que tiene para una persona sentirse capaz y segura en relación con las decisiones que toma y con sus comportamientos. Es el resultado de elementos como la autoestima, la percepción de control y dominio, así como de la experiencia que se ha tenido con anterioridad. La confianza se caracteriza por ser multidimensional y por constar de múltiples niveles. Así, se pueden diferenciar dos factores: por un lado, la confianza en las decisiones que se toman, considerada como la habilidad para tomar decisiones efectivas a la hora de adquirir y usar información; y, por otro lado, la protección definida como la capacidad para protegerse de engaños y situaciones de injusticia (Bearden et al., 2001).

Un concepto muy relacionado con la confianza es la idea de justicia. En este sentido, cabe señalar que la justicia se considera una condición necesaria para que haya confianza, de modo que cuando se percibe justicia esta percepción puede que tenga una influencia positiva sobre la confianza. La relación que se establece entre justicia y confianza es fundamental en las empresas de servicios, debido a que los productos que ofrecen se caracterizan por ser intangibles y difíciles de evaluar y donde los consumidores se guían por la confianza (Seiders y Berry, 1998).

Además, la confianza es una variable que adquiere más relevancia en el canal online que en el canal tradicional debido a que los consumidores toman sus decisiones de compra online guiados casi siempre por la confianza (Urban et al., 2000). Anderson y Srinivasan (2003) destacan que la confianza adquiere mucha relevancia en el entorno online debido al mayor riesgo que supone realizar las compras en dicho entorno.

Garbarino y Lee (2003) realizaron un estudio en Internet para analizar las reacciones de los consumidores ante la utilización de precios dinámicos y su influencia sobre la confianza. Los resultados muestran que tal y como ya se había puesto de manifiesto en otros estudios (Dickson y Kalapurakal, 1994; Kahneman et al., 1986a), en general los consumidores no suelen aceptar las variaciones en los precios que se establecen según la demanda, considerándose como injustos e influyendo de manera negativa sobre la confianza.

Así pues, cuando los consumidores perciben los precios como injustos, disminuye su percepción de justicia y, por consiguiente, su confianza en el vendedor (Monroe y Xia, 2006). Maxwell (2008) considera que en el proceso de decisión de un precio justo la confianza es un elemento clave. De modo que, la percepción de justicia que tiene el consumidor acerca de los precios va a determinar su comportamiento futuro condicionado por la confianza en el vendedor.

Normalmente, la confianza es un elemento que juega un papel importante a la hora de mantener una relación a largo plazo (Garbarino y Johnson, 1999), demostrándose que la confianza influye positivamente sobre la satisfacción del cliente (Benton y Maloni, 2005). 
A pesar de que los trabajos que se han encontrado se centran en analizar la relación entre percepción de justicia de precios y confianza en el vendedor, tomando como base autores como Seiders y Berry (1998) y Monroe y Xia (2006) se puede considerar también, extendiendo estos fundamentos, que la percepción de justicia de precios tiene efectos directos sobre la confianza a la hora de tomar decisiones por parte del individuo. De este modo, se plantea la siguiente hipótesis:

\section{H1: La percepción de justicia en el precio influirá directamente sobre la confianza en la decisión}

\subsection{Percepción de justicia de precios y lealtad}

La lealtad se puede definir de forma sencilla como el deseo de volver a comprar (Czepiel y Gilmore, 1987). Esta variable adquiere gran importancia para las empresas, sobre todo en el entorno virtual, ya que los clientes fieles son los más rentables (Reichheld et al., 2000).

El hecho de que cada vez haya más competencia conduce a que las empresas intenten mejorar y mantener la lealtad de sus clientes (Bruhn y Grund, 2000). Berry y Parasuraman (1991) establecen que para estimular la lealtad conductual se pueden diferenciar tres niveles: financiero, social y tácticas de vínculo estructural. De igual modo, Christy et al., (1996) han mostrado como las relaciones de vínculo ayudan a mejorar la lealtad conductual del cliente. Además, algunos autores (Ranaweera y Neely, 2003) señalan que la satisfacción tiene un efecto positivo a la hora de retener al cliente.

Dentro de la lealtad se pueden diferenciar dos tipos: conductual y actitudinal. La lealtad conductual se refiere a la experiencia que se ha tenido previamente, mientras que la lealtad actitudinal hace alusión al comportamiento futuro (Zins, 2001). Auh et al. (2007) se refieren a lealtad actitudinal como a las intenciones de permanecer y de compromiso con la organización. Mientras que la lealtad conductual se refiere más bien a un comportamiento efectivo de repetición de las compras. Así, la lealtad actitudinal se centra más en el compromiso del individuo en relación al comportamiento que va a tener en el futuro ya sea en relación con una marca o con un proveedor de servicios.

La utilización de un concepto u otro de lealtad depende del tipo de producto que se trate. Así pues, en productos de consumo frecuente se utiliza lealtad conductual, mientras que para productos de compra esporádica y servicios es conveniente utilizar como medidas de lealtad ambas dimensiones (conductual y actitudinal) (Moliner y Berenguer, 2010).

La lealtad puede vincularse a elementos como la comunicación boca-oído (Word of mouth) o las intenciones de volver a comprar (Söderlund, 2006). Además, distingue dos dimensiones dentro del concepto de lealtad: lealtad como comportamiento y lealtad como un estado mental (Dick y Basu, 1994; Rundle-Thiele y Mackay, 2001). El comportamiento de lealtad incluye, aspectos relacionados con las características del cliente (Baumann et al., 2005), la frecuencia de visitas (Bolton et al., 2000) y la duración de la relación (Rundle-Thiele y Mackay, 2001). Por otro lado, cabe señalar que la lealtad mental se refiere a la lealtad actitudinal e incluye elementos relacionados con actitudes (Dick y Basu, 1994), preferencias (Rundle-Thiele y Mackay, 2001) e intenciones como por ejemplo recomendaciones a terceros (Zeithaml et al., 1996).

En esta investigación, se ha optado por considerar la influencia que ejerce la percepción de la justicia del precio sobre la lealtad actitudinal dado que el objetivo es conocer el efecto de la percepción de justicia del precio sobre el comportamiento de compra futuro. Por ello, se van a considerar elementos como la recomendación positiva a otras personas boca-oído y las intenciones de volver a comprar.

Respecto a la relación establecida entre ambos contructos investigaciones previas, concretamente en el sector de servicios, ponen de manifiesto que la justicia percibida en el precio influye positivamente sobre la lealtad. Martín-Consuegra et al. (2007) concluyen con esta afirmación tras analizar los datos derivados de una encuesta personal a pasajeros de avión. Otros como Vázquez et al. (2007) también llegan a la misma conclusión tras realizar un estudio sobre la percepción de justicia relacionada con la recuperación del servicio en el caso de usuarios de teléfonos móviles. Por tanto, se establece la siguiente hipótesis:

\section{H2: La percepción de justicia del precio influirá directamente sobre la lealtad}

\subsection{Percepción de justicia de precios y satisfacción con el precio}

La satisfacción desde el punto de vista del consumidor tiene lugar cuando se cumplen o superan las expectativas previas a la compra. Asimismo, la satisfacción hace alusión a un estado emocional que tiene lugar como resultado de la interacción del cliente con el proveedor de un servicio (Crosby et al., 1990).

Cabe señalar que la satisfacción es un concepto que está muy relacionado con el concepto de justicia (Ordóñez et al., 2000). La percepción de mayor justicia en los precios puede que tenga como resultado que el consumidor perciba el precio como un precio aceptable y, por tanto, tenga una mayor satisfacción con el precio. 
Zielke (2008: 336) define satisfacción con el precio como "una reacción afectiva, resultado de la interacción de procesos mentales cognitivos y afectivos, que son causados y activados por experiencias específicas que tienen lugar ante las diferentes dimensiones de la percepción del precio". Asimismo, considera las dimensiones de percepción del precio (valor del dinero; nivel de precios; justicia en el precio; perceptibilidad de precios entendida como la facilidad con la que los precios se identifican en un establecimiento; procesamiento del precio que es la facilidad para procesar precios cuando se comparan distintos precios; ofertas especiales (e.g. descuentos); publicidad de precios y la categoría del rango de precios) como antecedentes de la satisfacción con el precio. Estas dimensiones pueden actuar favoreciendo la satisfacción con el precio o por el contrario, en el caso de la justicia en el precio, desfavoreciendo la satisfacción con el mismo.

La satisfacción con el precio en algunos estudios se considera como un constructo multidimensional. Matzler et al. (2006) establecen que las distintas dimensiones que constituyen la satisfacción con el precio, influyen sobre la satisfacción global del cliente, así como sobre su comportamiento a lo largo del tiempo. Según Matzler et al. (2006) la satisfacción con el precio es un constructo multidimensional, formado por dimensiones como la transparencia en el precio; la relación precio-calidad; el precio relativo; la confianza en el precio; la fiabilidad en el precio y la justicia en el precio.

En cambio, otros estudios se centran sólo en una dimensión de la satisfacción con el precio. Tal es el caso de Campbell (1999), que analiza cómo influye la justicia del precio sobre la percepción del precio; el efecto que tiene la relación precio-calidad (Fornell et al., 1996) o el efecto que tiene la percepción del precio sobre la satisfacción y comportamiento (Varki y Colgate, 2001).

Por otra parte, una de las relaciones que más se suele establecer con respecto a la percepción de injusticia es la insatisfacción, siendo la insatisfacción una experiencia negativa que está correlacionada con el malestar que despierta en el consumidor una situación de injusticia (Storm y Storm, 1987).

La percepción de injusticia influye tanto en el valor percibido del producto como en la satisfacción del consumidor, así como en las reacciones que se derivan como resultado de percepciones injustas que conducen a emociones negativas contra el vendedor (Xia et al., 2004).

En general, los consumidores tienden a comparar los precios cuando encuentran distintos precios para un mismo producto, comparaciones que puede que deriven en percepciones injustas del precio (Sinha, 2000), lo que conlleva que los clientes estén menos satisfechos con el precio que pagan (Bolton y Lemon, 1999).

Según Oliver y Swan (1989) la percepción de justicia del precio influye sobre la satisfacción del consumidor. Sinha y Batra (1999) establecen que la conciencia del precio y la satisfacción del consumidor se forman sobre la base de percepción de justicia de los precios. Así, cuando se perciben los precios como justos los consumidores experimentan mayor satisfacción (Ordóñez et al., 2000). Bei y Chiao (2001) observan que existe una relación positiva entre la justicia percibida en el precio y la satisfacción en el caso de servicios. Igualmente, Bolton et al. (2003) establecen que la percepción de justicia tiene un efecto directo y positivo sobre la satisfacción en el precio. Kauffman et al. (2010) muestran como la percepción de justicia tiene un efecto positivo sobre la satisfacción en el precio.

Hay que tener en cuenta que la percepción del precio puede tener lugar antes de la compra o después de la misma. En este sentido, algunos autores como Voss et al. (1998) establecen que dicha percepción condiciona la satisfacción del consumidor en el caso concreto de servicios. Bolton y Lemon (1999) realizan un estudio sobre dos servicios (comunicación y entretenimiento) para conocer cómo influye la satisfacción con el precio por parte del cliente en la satisfacción en general. Los resultados les muestran que la satisfacción con el precio tiene un impacto positivo sobre la satisfacción con el servicio aportado. Asimismo, Singh y Sirdeshmukh (2000) señalan que la justicia de precios constituye uno de los factores que determinan la satisfacción del consumidor. Del mismo modo, Martín-Consuegra et al. (2007) analizan, en el caso de los billetes de avión, la influencia que ejerce la percepción de justicia sobre la satisfacción y observan que ambas están relacionadas positivamente. Utilizando este marco contextual se formula la siguiente hipótesis:

H3: La percepción de justicia del precio tendrá un efecto directo sobre la satisfacción en el precio.

\section{Metodología}

El problema a investigar del presente trabajo consiste en analizar los efectos que la percepción de la justicia en el precio tiene sobre el comportamiento de compra del consumidor. Así pues, se considera la influencia que la percepción de justicia en el precio tiene sobre la confianza en la decisión de compra, 
la lealtad y la satisfacción del cliente. En este epígrafe se recogen las características de la investigación que definen la información obtenida, así como la herramienta y el procedimiento utilizado para la contrastación empírica.

\subsection{Muestra}

El método de recogida de información ha consistidito en una encuesta online autoadministrada a un panel de 600 usuarios que se enfrentaron a la decisión de reservar una habitación de hotel en un contexto simulado de cinco hoteles con estrategias de precios diferentes. El cuestionario se estructura en cuatro bloques. En un primer bloque se recogen cuestiones relativas a datos demográficos (sexo y edad), y aspectos tales como experiencia de reserva de alojamiento online con el fin de conocer sus hábitos en reservas de hoteles, conocimiento de precios, y precios de referencia que posee. El segundo y tercer bloque tienen lugar después realizar la reserva de hotel, y recogen preguntas relacionadas con justicia percibida y futuras intenciones. Por último, el cuarto bloque incluye preguntas socio-demográficas (estado civil, situación laboral, nivel de estudios, comunidad autónoma y nivel de ingresos). Finalmente, la muestra obtenida ha sido de 541 cuestionarios, resultado de eliminar aquellos cuya respuesta no se ajustaba a lo solicitado.

En cuanto a la selección de la muestra de individuos se ha realizado considerando cuotas a partir de la información publicada por el INE (2011) en su "Encuesta sobre equipamiento y Uso de Tecnologías de la Información y Comunicación en los Hogares" que recoge el perfil sociodemográfico de internautas con edades comprendidas entre 16 y 74 años, considerando aquellos que han comprado alguna vez a través de Internet. La tabla 1 muestra la ficha técnica de la investigación.

\section{Tabla 1: Ficha técnica de la investigación}

\begin{tabular}{|c|c|}
\hline Universo & Internautas que han comprado alguna vez en Internet \\
\hline Ámbito geográfico & España \\
\hline Tamaño muestral & 541 \\
\hline Fecha de trabajo de campo & 29 de Febrero a 27 de Marzo 2012 \\
\hline Tipo de estudio & Cuantitativo \\
\hline $\begin{array}{c}\text { Técnica de recogida de } \\
\text { información }\end{array}$ & Encuesta on-line \\
\hline Procedimiento de muestreo & Por cuotas \\
\hline Error Muestral (95\%) & $\pm 4 \%$ \\
\hline
\end{tabular}

Fuente: Elaboración propia

\subsection{Variables}

Las escalas utilizadas en las distintas variables latentes que comprende nuestro modelo de investigación y que nos van a permitir analizar las hipótesis establecidas constituyen un aspecto importante que merece especial atención.

En justicia percibida en el precio (JPP) se ha utilizado tanto la escala como los ítems que establecen Martin et al. (2009), pero los ítems se han adaptado a nuestro estudio. Las variables que aparecen en la tabla 2 recogen la media de seis ítems que hacen referencia a justicia distributiva y justicia procedimental en cada tipo de estrategia de gestión de demanda. Así pues, JPP1 recoge la media de justicia distributiva y procedimental en la estrategia de gestión de demanda con restricciones, JPP2 se refiere a la justicia distributiva y procedimental en la estrategia de gestión de demanda según el tiempo, JPP3 recoge los ítems relativos a justicia distributiva y procedimental en la estrategia de gestión de demanda según la ubicación; JPP4 que engloba los ítems de justicia distributiva en función de la estrategia de gestión de demanda según el número de noches; y, por último, JPP5 que está formado por los ítems de justicia distributiva y procedimental en la estrategia de gestión de demanda según anticipación. 
Tabla 2: Variables relacionadas con justicia percibida en el precio (JPP)

\begin{tabular}{|c|c|c|c|c|}
\hline Variable & \multicolumn{2}{|l|}{ Descripción } & Medida & Fuente \\
\hline JPP1 & $\begin{array}{l}\text { El precio más bajo que paga el cliente } \\
\text { por no poder cancelar la reserva es: } \\
\text { El proceso de fijación de precios que se } \\
\text { sigue a la hora de establecer precios } \\
\text { más bajos para aquellos que no pueden } \\
\text { modificar o cancelar la reserva me } \\
\text { parece: }\end{array}$ & $\begin{array}{l}\text { - justo } \\
\text { - razonable } \\
\text { - aceptable } \\
\text { - justo. } \\
\text { - razonable. } \\
\text { - aceptable. }\end{array}$ & $\begin{array}{c}\text { Escala } \\
\text { Likert de } 7 \\
\text { puntos (1 } \\
\text { totalmente } \\
\text { en } \\
\text { desacuerdo- } \\
7 \\
\text { totalmente } \\
\text { de acuerdo) }\end{array}$ & $\begin{array}{c}\text { Adaptados } \\
\text { de Martin } \\
\text { et al. (2009) }\end{array}$ \\
\hline JPP2 & $\begin{array}{l}\text { El precio de la habitación los viernes y } \\
\text { sábados es: } \\
\text { El proceso de fijación de precios que se } \\
\text { sigue a la hora de establecer precios } \\
\text { más altos los viernes y sábados me } \\
\text { parece: }\end{array}$ & $\begin{array}{l}\text { - justo } \\
\text { - razonable } \\
\text { - aceptable } \\
\text { - justo. } \\
\text { - razonable. } \\
\text { - aceptable }\end{array}$ & & \\
\hline JPP3 & $\begin{array}{l}\text { El precio de la habitación con vistas es: } \\
\text { El proceso de fijación de precios que se } \\
\text { sigue a la hora de establecer un precio } \\
\text { más alto para la habitación con vistas } \\
\text { me parece: }\end{array}$ & $\begin{array}{l}\text { - justo } \\
\text { - razonable } \\
\text { - aceptable } \\
\text { - justo. } \\
\text { - razonable. } \\
\text { - aceptable. }\end{array}$ & & \\
\hline JPP4 & $\begin{array}{l}\text { El precio más bajo a partir de la cuarta } \\
\text { noche es: } \\
\text { El proceso de fijación de precios que se } \\
\text { sigue a la hora de establecer un precio } \\
\text { más bajo conforme se aumenta el } \\
\text { número de noches me parece: }\end{array}$ & $\begin{array}{l}\text { - justo } \\
\text { - razonable } \\
\text { - aceptable } \\
\text { - justo. } \\
\text { - razonable. } \\
\text { - aceptable. }\end{array}$ & & \\
\hline JPP5 & $\begin{array}{l}\text { El precio más bajo que paga el cliente } \\
\text { que realiza su reserva con anticipación } \\
\text { es } \\
\text { El proceso de fijación de precios que se } \\
\text { sigue a la hora de establecer precios } \\
\text { más bajos para aquellos que reservan } \\
\text { con anticipación me parece: }\end{array}$ & $\begin{array}{l}\text { - justo } \\
\text { - razonable } \\
\text { - aceptable } \\
\text { - justo. } \\
\text { - razonable. } \\
\text { - aceptable. }\end{array}$ & & \\
\hline
\end{tabular}

Fuente: Elaboración propia

Para medir la confianza en la decisión hemos utilizados los ítems de los distintos niveles que comprende que son: adquisición y procesamiento de información; formación del conjunto a considerar, y, por último, resultados personales y sociales (Bearden et al., 2001), utilizando una escala likert de 7 puntos (Chelminski y Coulter, 2007). 
Tabla 3: Variables relacionadas con confianza en la decisión (CD)

\begin{tabular}{|c|c|c|c|}
\hline Variable & Descripción & Medida & Fuente \\
\cline { 1 - 2 } CD1 & Tengo confianza con la decisión tomada & \\
\cline { 1 - 2 } CD2 & No me ha costado mucho decidir & $\begin{array}{c}\text { Escala Likert } \\
\text { de 7 puntos (1 } \\
\text { totalmente en } \\
\text { desacuerdo- } 7 \\
\text { totalmente de } \\
\text { acuerdo) }\end{array}$ & $\begin{array}{c}\text { Adaptado de } \\
\text { Bearden } \text { et al. } \\
\text { (2001) }\end{array}$ \\
\cline { 1 - 2 } CD3 & Creo que he sido capaz de identificar la mejor para mí & \\
\hline CD5 & Creo que he sido capaz de recoger toda la \\
información relevante & He tomado la decisión correcta & \\
\hline CD6 & He identificado rápidamente la mejor opción & \\
\hline
\end{tabular}

Fuente: Elaboración propia

Aunque algunos autores han distinguido tres dimensiones dentro de la lealtad (boca-oído, tolerancia al precio e intenciones de volver a comprar). En nuestro caso, al igual que Söderlund (2006), nos hemos centrado en las dimensiones: boca-oído e intenciones de compra. Para medir la lealtad, hemos utilizado los ítems y escalas recogidos en la tabla 4.

Tabla 4: Variables relacionadas con lealtad (L)

\begin{tabular}{|c|c|c|c|}
\hline Variable & Descripción & Medida & Fuente \\
\hline L1 & Recomendaría el hotel que he elegido & \multirow{4}{*}{$\begin{array}{l}\text { Escala Likert } \\
\text { de } 7 \text { puntos (1 } \\
\text { totalmente en } \\
\text { desacuerdo- } 7 \\
\text { totalmente de } \\
\text { acuerdo) }\end{array}$} & \multirow{2}{*}{$\begin{array}{l}\text { Adaptado de Zeithaml et al. } \\
\text { (1996); Söderlund (2006); } \\
\text { Vázquez et al. (2007); Cater } \\
\text { y Zabkar (2009). }\end{array}$} \\
\hline L2 & $\begin{array}{l}\text { Si mis amigos o familiares estuvieran } \\
\text { buscando, les recomendaría esta decisión }\end{array}$ & & \\
\hline L3 & $\begin{array}{c}\text { Si tuviera que elegir otra vez, volvería a } \\
\text { elegir este hotel }\end{array}$ & & \multirow{2}{*}{$\begin{array}{l}\text { Adaptado de Zeithaml et } \\
\text { al. (1996); Vázquez et al. } \\
\text { (2007) }\end{array}$} \\
\hline L4 & $\begin{array}{c}\text { Aunque otros me ofrezcan precios más } \\
\text { bajos creo que continuaría eligiendo este } \\
\text { hotel }\end{array}$ & & \\
\hline
\end{tabular}

Fuente: Elaboración propia

Dentro de satisfacción nos hemos centrado en satisfacción con el precio, usando escalas e ítems adaptados de estudios anteriores que aparecen recogidos en la tabla 5.

Tabla 5: Variables relacionadas con satisfacción con el precio (SP)

\begin{tabular}{|c|c|c|c|}
\hline Variable & Descripción & Medida & Fuente \\
\hline SP1 & $\begin{array}{c}\text { En general, estoy satisfecho con la compra que } \\
\text { he realizado }\end{array}$ & \multirow{6}{*}{$\begin{array}{l}\text { Escala Likert } \\
\text { de } 7 \text { puntos ( } 1 \\
\text { totalmente en } \\
\text { desacuerdo- } 7 \\
\text { totalmente de } \\
\text { acuerdo) }\end{array}$} & \multirow{6}{*}{$\begin{array}{l}\text { Adaptado de Voss et } \\
\text { al. (1998); Ordóñez et } \\
\text { al. (2000); Cao et al. } \\
\text { (2003); Kauffman et al. } \\
\text { (2010) }\end{array}$} \\
\hline SP2 & $\begin{array}{c}\text { Estoy satisfecho con el precio pagado por la } \\
\text { habitación }\end{array}$ & & \\
\hline SP3 & $\begin{array}{l}\text { Creo que he conseguido las mejores condiciones } \\
\text { posibles con el precio pagado }\end{array}$ & & \\
\hline SP4 & Estoy contento con el precio pagado & & \\
\hline SP5 & $\begin{array}{c}\text { El precio pagado hace que sienta que el } \\
\text { producto es barato }\end{array}$ & & \\
\hline SP6 & $\begin{array}{l}\text { El precio pagado hace que me sienta bien con } \\
\text { mi compra }\end{array}$ & & \\
\hline
\end{tabular}

Fuente: Elaboración propia 


\subsection{Análisis Factorial Exploratorio}

Teniendo en cuenta las características de la información hemos optado por estimar el modelo mediante mínimos cuadrados parciales (PLS). Para ello, en primer lugar se ha desarrollado un análisis factorial exploratorio (AFE) que ha permitido comprobar que los ítems elegidos presentan un buen comportamiento como indicadores de cada una de las variables latentes. En la escala justicia percibida en el precio los resultados ponen de manifiesto que los ítems que la forman cargan en un sólo factor. Este factor explica el 56,08\% de la varianza. El test de esfericidad de Bartlett es significativo, y además la prueba de adecuación muestral de la KMO y el alpha de Cronbach están próximos a 1.

\section{Tabla 6: AFE: justicia percibida en el precio}

\begin{tabular}{|l|c|}
\hline \multicolumn{2}{|c|}{ Justicia percibida en el precio } \\
\hline $\begin{array}{l}|c| \\
\text { KMO: } 0,829 \\
\text { Prueba de esfericidad de Barlett (Valor } \\
\text { Chi-cuadrado): } 769,044 \\
\text { p-valor: } 0,000\end{array}$ & Componente \\
Varianza total explicada: 56,084 & 1 \\
Alpha de Cronbach: 0,801 & $\mathbf{0 , 7 9 4}$ \\
\hline Estrategia de gestión de demanda según restricciones-cancelación (JPP1) & $\mathbf{0 , 8 7 8}$ \\
\hline Estrategia de gestión de demanda según la ubicación (JPP2) & $\mathbf{0 , 9 0 4}$ \\
\hline Estrategia de gestión de demanda según el tiempo (JPP3) & $\mathbf{0 , 8 5 5}$ \\
\hline Estrategia de gestión de demanda según el número de noches (JPP4) & $\mathbf{0 , 8 3 6}$ \\
\hline Estrategia de gestión de demanda según restricciones-anticipación (JPP5) & \\
\hline
\end{tabular}

Fuente: Elaboración propia

Con respecto a la dimensión de lealtad los resultados ponen de manifiesto que todos los ítems cargan en un único factor, así como también se observa que tanto el test de esfericidad de Bartlett, como la prueba de adecuación muestral de la KMO, y el Alpha de Cronbach son satisfactorios. Este análisis tiene como resultado un factor que explica el $71,8 \%$ de la varianza.

\section{Tabla 7: AFE: lealtad}

\begin{tabular}{|c|c|}
\hline \multicolumn{2}{|l|}{ Lealtad } \\
\hline $\begin{array}{l}\text { KMO: } 0,733 \\
\text { Prueba de esfericidad de Barlett (Valor } \\
\text { Chi-cuadrado): } 1462,142 \\
\text { p-valor: } 0,000\end{array}$ & Componente \\
\hline $\begin{array}{l}\text { Varianza total explicada: } 71,788 \\
\text { Alpha de Cronbach: } 0,856\end{array}$ & 1 \\
\hline Recomendaría el hotel que he elegido (L1) & 0,916 \\
\hline Si mis amigos o familiares estuvieran buscando, les recomendaría esta decisión (L2) & 0,908 \\
\hline Si tuviera que elegir otra vez, volvería a elegir este hotel (L3) & 0,837 \\
\hline $\begin{array}{l}\text { Aunque otros me ofrezcan precios más bajos creo que continuaría eligiendo este } \\
\text { hotel (L4) }\end{array}$ & 0,712 \\
\hline
\end{tabular}

Fuente: Elaboración propia

En relación con la escala satisfacción con el precio todos los ítems cargan en un solo factor. Este factor explica el 74\% de la varianza. Además, el test de esfericidad de Bartlett es significativo, y la prueba de adecuación muestral de la KMO, así como el Alpha de Cronbach están muy próximos a 1. 
Tabla 8: AFE: satisfacción con el precio

\begin{tabular}{|l|c|}
\hline \multicolumn{2}{|c|}{ Satisfacción con el precio } \\
\hline $\begin{array}{l}\text { KMO: 0,897 } \\
\text { Prueba de esfericidad de Barlett (Valor } \\
\text { Chi-cuadrado): } 2636,870 \\
\text { p-valor: 0,000 }\end{array}$ & Componente \\
$\begin{array}{l}\text { Varianza total explicada: } 74,014 \\
\text { Alpha de Cronbach: 0,928 }\end{array}$ & 1 \\
\hline En general, estoy satisfecho con la compra que he realizado (SP1) & $\mathbf{0 , 8 5 0}$ \\
\hline Estoy satisfecho con el precio pagado por la habitación (SP2) & $\mathbf{0 , 9 0 2}$ \\
\hline Creo que he conseguido las mejores condiciones posibles con el precio pagado (SP3) & $\mathbf{0 , 8 5 5}$ \\
\hline Estoy contento con el precio pagado (SP4) & $\mathbf{0 , 9 1 6}$ \\
\hline El precio pagado hace que sienta que el producto es barato (SP5) & $\mathbf{0 , 7 3 3}$ \\
\hline El precio pagado hace que me sienta bien con mi compra (SP6) & $\mathbf{0 , 8 9 3}$ \\
\hline
\end{tabular}

Fuente: Elaboración propia

Por último, señalar que en la escala de confianza en la decisión inicialmente se han descartado dos ítems, ya que más que medir la confianza en la decisión, estarían midiendo la desconfianza. Una vez eliminados, los resultados muestran un único factor que explica el 67,03\% de la varianza, y presenta un Alpha de Cronbach próximo a 1. En cuanto a la adecuación de este análisis queda comprobada mediante la prueba de esfericidad de Bartlett, y la prueba de adecuación muestral KMO.

Tabla 9: AFE: confianza en la decisión

\begin{tabular}{|l|c|}
\hline \multicolumn{1}{|c|}{ Confianza en la decisión } \\
\hline $\begin{array}{l}\text { KMO: 0,884 } \\
\text { Prueba de esfericidad de Barlett (Valor } \\
\text { Chi-cuadrado): } 1890,478 \\
\text { p-valor: 0,000 }\end{array} \begin{array}{c}\text { Carianza total explicada: } 67,031 \\
\text { Alpha de Cronbach: 0,9 }\end{array}$ & 1 \\
\hline Tengo confianza con la decisión tomada (CD1) & $\mathbf{0 , 8 2 6}$ \\
\hline No me ha costado mucho decidir (CD2) & $\mathbf{0 , 7 2 8}$ \\
\hline Creo que he sido capaz de identificar la mejor opción para mí (CD3) & $\mathbf{0 , 8 4 4}$ \\
\hline Creo que he sido capaz de recoger toda la información relevante (CD4) & $\mathbf{0 , 7 9 0}$ \\
\hline He tomado la decisión correcta (CD5) & $\mathbf{0 , 8 6 6}$ \\
\hline He identificado rápidamente la mejor opción para mí (CD6) & $\mathbf{0 , 8 5 1}$ \\
\hline
\end{tabular}

Fuente: Elaboración propia

\subsection{Modelo de ecuaciones estructurales}

Considerando los ítems seleccionados con el análisis factorial exploratorio se ha construido y estimado el modelo que aparece recogido en la figura 1 . 
Figura 1: Estimación del modelo de ecuaciones estructurales

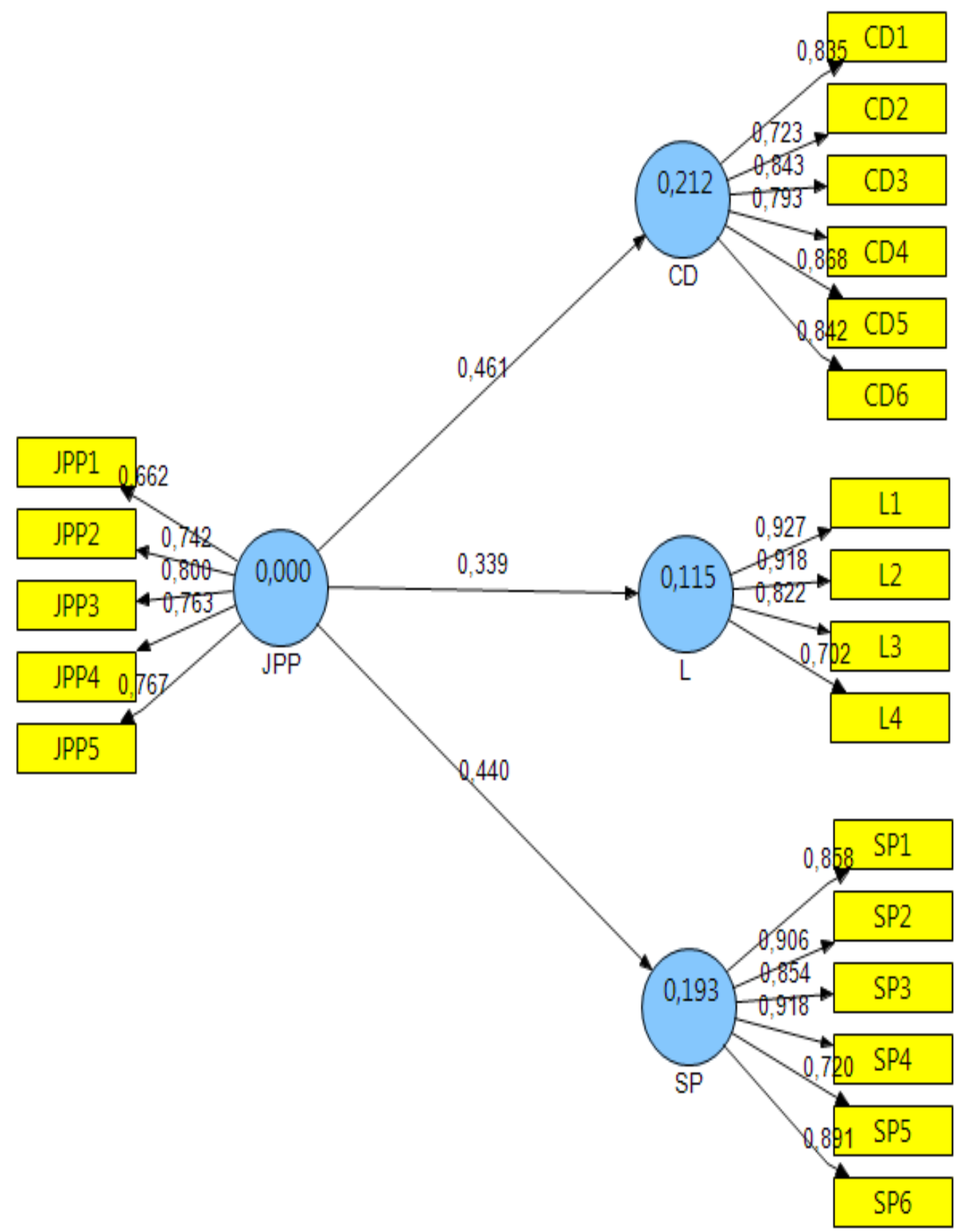


Fuente: Elaboración propia

El proceso de estimación se ha desarrollado usando el software SmartPLS 2.0.M3 (www.smartpls.de). La tabla 10 ofrece los resultados de la evaluación de la fiabilidad y validez convergente. Los resultados muestran que todos los indicadores son significativos y sus cargas estandarizadas mayores de 0,60 (Bagozzi y Yi, 1988). La fiabilidad del instrumento de medida es buena ya que, en todos los casos, el alfa de Cronbach supera la recomendación de Nunnally y Bernstein (1994) de 0,70; y los índices de fiabilidad compuesta y la varianza extraída promedio (AVE) superan para todos los factores los valores recomendados de 0,8 y 0,5 , respectivamente.

Tabla 10: Validación del instrumento de medida. Fiabilidad y validez convergente.

\begin{tabular}{|c|c|c|c|c|c|c|}
\hline Factor & Indicador & Carga & $\begin{array}{c}\text { Valor t } \\
\text { (Bootstrap) }\end{array}$ & $\begin{array}{l}\text { Alpha de } \\
\text { Cronbach }\end{array}$ & $\begin{array}{l}\text { Fiabilidad } \\
\text { Compuesta }\end{array}$ & AVE \\
\hline \multirow{5}{*}{$\begin{array}{l}\text { Justicia } \\
\text { percibida } \\
\text { (JPP) }\end{array}$} & JPP1 & $0,662^{* *}$ & 8,3175 & \multirow{5}{*}{0,8031} & \multirow{5}{*}{0,9239} & \multirow{5}{*}{0,5602} \\
\hline & JPP2 & $0,742^{* *}$ & 15,2658 & & & \\
\hline & JPP3 & $0,800^{* * *}$ & 18,2118 & & & \\
\hline & JPP4 & $0,763^{* *}$ & 17,6048 & & & \\
\hline & JPP5 & $0,767^{* *}$ & 17,0448 & & & \\
\hline \multirow{6}{*}{$\begin{array}{l}\text { Confianza en } \\
\text { la decisión } \\
\text { (CD) }\end{array}$} & CD1 & $0,835^{* *}$ & 26,7892 & \multirow{6}{*}{0,9008} & \multirow{6}{*}{0,8639} & \multirow{6}{*}{0,670} \\
\hline & CD2 & $0,723^{* *}$ & 12,5816 & & & \\
\hline & CD3 & $0,843^{* *}$ & 21,9992 & & & \\
\hline & CD4 & $0,793^{* *}$ & 18,2497 & & & \\
\hline & CD5 & $0,868^{* *}$ & 28,7816 & & & \\
\hline & CD6 & $0,842^{* *}$ & 26,5395 & & & \\
\hline \multirow{4}{*}{ Lealtad (L) } & L1 & $0,927 * *$ & 13,1849 & \multirow{4}{*}{0,8656} & \multirow{4}{*}{0,9094} & \multirow{4}{*}{0,717} \\
\hline & L2 & $0,918^{* *}$ & 13,3123 & & & \\
\hline & L3 & $0,822^{* *}$ & 11,6991 & & & \\
\hline & $\mathrm{L4}$ & $0,702^{* *}$ & 11,117 & & & \\
\hline \multirow{6}{*}{$\begin{array}{c}\text { Satisfacción } \\
\text { con el precio } \\
\text { (SP) }\end{array}$} & SP1 & $0,858^{* *}$ & 32,5241 & \multirow{6}{*}{0,9286} & \multirow{6}{*}{0,9443} & \multirow{6}{*}{0,7399} \\
\hline & SP2 & $0,906^{* *}$ & 54,7018 & & & \\
\hline & SP3 & $0,854^{* *}$ & 32,1712 & & & \\
\hline & SP4 & $0,918^{* *}$ & 56,621 & & & \\
\hline & SP5 & $0,720 * *$ & 15,9363 & & & \\
\hline & SP6 & $0,891^{* *}$ & 42,1666 & & & \\
\hline
\end{tabular}

Fuente: Elaboración propia ** $p<0,01$

También se cumple el criterio de validez discriminante (Fornell y Larcker, 1981), ya que el AVE de cada par de factores es superior al cuadrado de la correlación estimada entre ellos:

Tabla 11. Correlaciones entre las variables latentes

\begin{tabular}{|c|c|c|c|c|}
\hline & CD & JPP & L & SP \\
\hline CD & 0,6700 & & & \\
\hline JPP & 0,2122 & 0,5602 & & \\
\hline L & 0,4635 & 0,1151 & 0,7173 & 0,7399 \\
\hline SP & 0,5714 & 0,1933 & 0,5506 & \\
\hline
\end{tabular}

Fuente: Elaboración propia 
La tabla 12 muestra los resultados del contraste de las hipótesis planteadas en este trabajo. De estos resultados hay que destacar que la percepción de justicia de precios influye de forma positiva y significativa sobre la confianza en la decisión y, por lo tanto, debemos aceptar la primera hipótesis de nuestro trabajo. En relación a la lealtad, existe una influencia significativa de la percepción de justicia sobre la misma, aceptando la hipótesis segunda, si bien, la relación es de menos intensidad que en los otros dos casos. En relación a la satisfacción, tal y como habíamos considerado en la hipótesis tres, ésta se ve afectada de forma significativa por la percepción de justicia de precios.

Tabla 12. Contraste de hipótesis

\begin{tabular}{|c|c|c|c|c|}
\hline Hipótesis & Relación & Coeficiente & $\begin{array}{c}\text { Valor t } \\
\text { (Bootstrap) }\end{array}$ & p-valor \\
\hline H1 & $\begin{array}{c}\text { Justicia percibida en el } \\
\text { precio } \rightarrow \text { confianza en la decisión }\end{array}$ & $0,461^{* *}$ & 6,349 & 0,000 \\
\hline H2 & $\begin{array}{c}\text { Justicia percibida en el } \\
\text { precio } \rightarrow \text { lealtad }\end{array}$ & $0,339^{* *}$ & 4,035 & 0,000 \\
\hline H3 & $\begin{array}{c}\text { Justicia percibida en el } \\
\text { precio } \rightarrow \text { satisfacción con el precio }\end{array}$ & $0,440^{* *}$ & 6,066 & 0,000 \\
\hline
\end{tabular}

Fuente: Elaboración propia ** $\mathrm{p}<0,01$

\section{Conclusiones y futuras líneas de investigación}

En los últimos años, ha aumentado la importancia que los consumidores otorgan a la percepción de justicia en los precios en sus decisiones de compra. Esto ha provocado que los vendedores muestren cada vez más un mayor interés por conocer los efectos que la percepción de precios puede desencadenar dado que los efectos de una percepción negativa suelen ser de gran intensidad y duración en el tiempo, si bien, la mayoría de ellos no se manifiestan en el corto plazo.

Ante esta situación, este trabajo ha permitido determinar que la percepción de justicia ejerce una influencia positiva y significativa sobre la confianza en la decisión, la lealtad y la satisfacción con el precio. Por lo tanto, una percepción negativa va a llevar a la consideración del precio como injusto lo que va a provocar, a largo plazo, una pérdida de clientela.

Dentro de las limitaciones de este trabajo hay que tener en cuenta que ha sido desarrollado bajo un entorno simulado online por lo que tal vez hay que ampliar ese entorno incluyendo un entorno físico y un entorno online real. Además, se ha valorado la percepción de justicia en un único servicio, la reserva de hotel, por lo que puede ser interesante analizar si ese comportamiento se repite en otras situaciones.

Estas limitaciones abren futuras líneas de investigación que pueden venir centradas fundamentalmente en dos aspectos; por un lado, el desarrollo de un modelo completo considerando los factores que condicionan la percepción de justicia y las consecuencias de la misma y, por otro lado, teniendo en cuenta la posibilidad de ampliar el análisis con otro tipo de productos y consumidores de otros países para comprobar si los resultados obtenidos se repiten o cambian de forma significativa.

\section{Bibliografia}

Anderson, R.E. \& Srinivasan, S.

2003. "E-satisfaction and e-loyalty: a contingency framework". Psychology \& Marketing, 20(2): 123-138. Andrés, M.E., Gómez, M.A. y Mondéjar, J.A.

2012. "Antecedentes en la percepción de justicia del precio: el caso de una reserve hotelera online".

XXIV Congreso Nacional de Marketing, Mallorca.

Auh, S., Bell, S.J., McLeod, C.S. y Shih, E.

2007. "Co-production and customer loyalty in financial services". Journal of Retailing, 83 (3): 359-370. Bagozzi, R.P. y Yi, T.

1988. "On the evaluation of structural equation models". Journal of the Academy of Marketing Science, 16 (1): 74-94. 
Baumann, C., Burton, S. y Elliot, G.

2005. "Determinants of customer loyalty and share of wallet in retail banking". Journal of Financial

Services Marketing, 9: 231-248.

Bearden, W.O., Hardesty, D. y Rose, R.

2001. "Consumer self-confidence: refinements in conceptualization and measurement". Journal of

Consumer Research, 28(1): 121-134.

Bei, L.T. y Chiao, Y.C.

2001. "An Integrated model for the effects of perceived product, perceived service quality". Journal of

Consumer Satisfaction, Dissatisfaction and Complaining Behavior, 14: 125-140.

Benton, W.C. y Maloni, M.

2005. "The influence of power driven buyer/seller relationships on supply chain satisfaction". Journal of Operations Management, 23(1): 1-22.

Berry, L.L. y Parasuraman, A.

1991. Marketing service-competing through quality. New York: The Free Press.

Bolton, R., Kannan, P.K. y Bramlett, M.D.

2000. "Implications of loyalty program membership and service experiences for customer retention and value". Journal of the Academy of Marketing Science, 28(1): 95-108.

Bolton, L.E.; Keh, H.T. y Alba, J.W.

2010. "How do price fairness perceptions differ across culture?". Journal of Marketing Research, 47 (junio): 564-576.

Bolton, R.N. y Lemon, K.N.

1999. "A dynamic model of customers'usage of services: usage as an antecedent and consequence". Journal of Marketing Research, 36(2): 171-186.

Bolton, L.E., Warlop, L. y Alba, J.E.

2003. "Consumer Perceptions of Price (Un) Fairness". Journal of Consumer Research, 29: 474-491.

Bruhn, M. y Grund, M.A.

2000. "Theory, development, and implementation of national customer satisfaction indices: the Swiss index of customer satisfaction (SWICS)". Total Quality Management, 11(7): 1017-1028.

Campbell, M.C.

1999. "Perceptions of price unfairness: antecedents and consequences". Journal of Marketing Research, 36(2): 187-199.

Cao, Y., Gruca, T.S. y Klemz, B.R.

2003. "Internet pricing, price satisfaction and customer satisfaction". International Journal of Electronic Commerce, 8(2): 31-50.

Cater, B. y Zabkar, V.

2009. "Antecedents and consequences of commitment in marketing research services: The client's perspective”. Industrial Marketing Management, 38: 785-795.

Chelminski, P. y Coulter, R.A.

2007. "On market mavens and consumer self-confidence: a cross-cultural study". Psychology \& Marketing, 24(1): 69-91.

Christy, R.; Oliver, G. y Penn, J.

1996. "Relationship marketing in consumer markets". Journal of Marketing Management, 12(1): 175-187.

Crosby, L.A., Evans, K.R. y Cowles, D.

1990. "Relationship quality in services selling: An interpersonal influence perspective". Journal of Marketing, 54(3): 68-81.

Czepiel, J.A. y Gilmore, R.

1987. "Exploring the Concept of Loyalty in Services". En . Czepiel, A. Congram, C.A. y Shanahan, J. (Eds.), The Services Marketing Challenge: Integrating for Competitive Advantage (pp. 91-94). Chicago: Amer Marketing Assn.

Dick, A.S. y Basu, K.

1994. "Customer loyalty: toward an integrated conceptual framework". Journal of the Academy of Marketing Science, 22: 99-113.

Dickson, P.R. y Kalapurakal, R.

1994. "The use and perceived price fairness of price-setting rules in the bulk electricity market". Journal of Economic Psychology, 15(3): 427-448.

Fornell, C., Johnson, M., Anderson, E.W., Cha, J. y Everitt, B.

1996. "The American customer satisfaction index: nature, purpose and findings". Journal of Marketing, 60: 7-18. 
Fornell, C. y Larcker, D.

1981. "Evaluating structural equations models with unobservable variables and measurement error". Journal of Marketing Research, 18: 39-50.

Garbarino, E. y Johnson, M.

1999. "The different roles of satisfaction, trust and commitment in customer relationships". Journal of Marketing, 63: 70-87.

Garbarino, E. y Lee, O.F.

2003. "Dynamic pricing in Internet retail: effects on consumer trust". Psychology \& Marketing, 20(6): 495-513.

Gielissen, R., Dutilh, C.E. y Graafland, J.J.

2008. "Perceptions of price fairness: an empirical research". Business \& Society, 47(3): 370-389.

Hosteltur

2011. "Noticias sobre turismo". Disponible en: http://www.hosteltur.com

INE

2011. "Encuesta sobre Equipamiento y Uso de Tecnologías de la Información y Comunicación en los hogares". Disponible en: http://www.ine.es

INE

2012. "Encuesta de Ocupación Hotelera". Disponible en: http://www.ine.es

Kahneman, D., Knetsch, J.L. y Thaler, R.H.

1986a. "Fairness and the assumptions of economics". Journal of Business, 59(4): 285-300.

Kahneman, D., Knetsch, J.L. y Thaler, R.H.

1986b. "Fairness as a constraint on profit seeking: entitlements in the market". The American Economic

Review, 76(4): 728-741.

Kauffman, R.J., Lai, H. y Ho, C.-T.

2010. "Incentive mechanisms, fairness and participation in online group-buying auctions". Electronic Commerce Research and Applications, 9: 249-262.

Lichtenstein, D.R., Ridgway, N.M. y Netemeyer, R.G.

1993. "Price perceptions and consumer shopping behaviour: A field study". Journal of Marketing Research, 30: 234-245.

Martin, W.C., Ponder, N. y Lueg, J.E.

2009. "Price fairness perceptions and customer loyalty in a retail context". Journal of Business Research, 62: 588-593.

Martín-Consuegra, D., Molina, A. y Esteban, A.

2007. "An integrated model of price, satisfaction and loyalty: an empirical analysis in the service sector". Journal of Product \& Brand Management, 16/7: 459-468.

Matzler, K., Würtele, A. y Renzl, B.

2006. "Dimensions of price satisfaction: a study in the retail banking industry". International Journal of Bank Marketing, 24(4): 216-231.

Maxwell, S.

2008. The price is wrong: understanding what makes a price seem fair and the true cost of unfair pricing. New Jersey: John Wiley \& Sons.

Moliner, B. y Berenguer, G.

2010. "La lealtad como base de segmentación de clientes en el comercio minorista". Tribuna de Economía, 855, ICE: 139-152.

Monroe, K.M y Xia, L.

2006. "The price is unfair! Reforming pricing management". En Sheth, J.N. y SISODIA, R.S. (Eds.), Does marketing need reform? (pp. 158-165). Nueva York: Sharpe.

Nunnally, J. y Bernstein, I.

1994. Psychometric Theory. Nueva York: McGraw Hill.

Oliver, R.L. y Swan, J.E.

1989. "Consumer perceptions of interpersonal equity and satisfaction in transactions: a filed survey approach”. Journal of Marketing, 53: 21-35.

ONTSI

2011. "Estudio sobre Comercio Electrónico B2C 2011". Disponible en: http://www.ontsi.red.es/ontsi Ordóñez, L.D., Connolly, T. y Coughlan R.

2000. "Multiple reference points in satisfaction and fairness assessment". Journal of Behavioral Decision Making, 13(3): 329-344. 
Ranaweera, C. y Neely, A.

2003. "Some moderating effects on the service quality-customer retention link: mobile phone service in France”. International Journal of operations and production management, 23(2): 230-248.

Reichheld, F.F., Markey, R.G. y Hopton, C.

2000. "E-customer loyalty-applying the traditional rules of business for online success". European Business Journal, 12(4): 173-179.

Rundle-Thiele, S. y Mackay, M.M.

2001. "Assessing the performance of brand loyalty measures". Journal of Services Marketing, 15(7): 529-546.

Seiders, K. y Berry, L.L.

1998. "Service Fairness: What it is and why it matters". The Academy of Management Executive, 12(2): 8-20.

Singh, J. y Sidershmukh, D.

2000. "Agency and trust mechanisms in consumer satisfaction and loyalty judgments". Journal of the Academy of Marketing Science, 28(1): 150-167.

Sinha, I.

2000. "Cost transparency: The net's real threat to prices and brands". Harvard Business Review, 78(2): 43-49.

Sinha, I. y Batra, R.

1999. "The effect of consumer price consciousness on private label purchase". International Journal of Research in Marketing, 16(3): 237-251.

Söderlund, M.

2006. "Measuring customer loyalty with multi-item scales". International Journal of Service Industry Management, 17(1): 76-98.

Storm, C. y Storm, T.

1987. "A taxonomic study of the vocabulary of emotions". Journal of Personality and Social Psychology, 53: 805-816.

Urban, G.L., Sultan F., y Quails, W.

2000. "Placing trust at the center of your Internet strategy". Sloan Management Review, 39-48. Disponible en: http://sloanreview.mit.edu

Varki, S y Colgate, M.

2001. The role of price perceptions in an integrated model of behavioral intentions. Journal of Service Research, 3(3): 232-240.

Vázquez, R., Díaz, A., Suárez, A. y Río, A.

2007. "Justicia percibida y satisfacción del cliente con la solución del problema como determinantes de la lealtad en contextos de fallo de servicio". XX Congreso anual de AEDEM. Disponible en: http:// dialnet.unirioja.es/servlet/fichero_articulo?codigo=2486883

Voss, G.B., Parasuraman, A. y Grewal, D.

1998. "The roles of price, performance, and expectations in determining satisfaction in service exchanges". Journal of Marketing, 62(4): 46-61.

Xia, L.; Monroe, K.B. y Cox, J.L.

2004. "The price is unfair!.A conceptual framework of price fairness perceptions". Journal of Marketing, 68: $1-15$

Yu, S.F.

2008. "Price perception of online airline ticket shoppers". Journal of Air Transport Management, 14(2): 66-69.

Zeithaml, V.A., Berry, L.L. y Parasuraman, A.

1996. "The behavioral consequences of service quality". Journal of Marketing, 60: 31-46.

Zielke, S.

2008. "Exploring asymmetric effects in the formation of retail price satisfaction". Journal of Retailing and Consumer Services, 15: 335-347.

Zins, A.H.

2001. "Relative attitudes and commitment in customer loyalty models". International Journal of Service Industry Management, 12(3): 269-294.

Recibido:

$21 / 05 / 2013$

Reenviado:

$02 / 09 / 2013$

Aceptado:

$15 / 10 / 2013$

Sometido a evaluación por pares anónimos 